\title{
Serum Level of Preptin in Children with Type-1 Diabetes Mellitus and Its Relation to Diabetic Nephropathy \\ Wafaa Fathy Mohamed Elsaeed ${ }^{1}$, Hadeel Mohamed Abdel Rahman', Nader Guima Sasi ${ }^{1}$, Haidy Essam Eldin Zidan ${ }^{2}$ \\ Departments of ${ }^{1}$ Pediatrics and ${ }^{2}$ Medical Biochemistry \& Molecular Biology, Faculty of Medicine - Zagazig University, Egypt. \\ *Corresponding author: Nader Guima Sasi, Mobile: (+20)01102916031, E-mail: narrabty@ gmail.com
}

\begin{abstract}
Background: Type 1 diabetes is one of the most prevalent long-term diseases of childhood globally.

Objective: This study aimed to evaluate a new biomarker such as preptin in patients with type 1 diabetes and to assess its relation to diabetic nephropathy.

Patients and methods: This study was a case-control study that carried on 34 children and adolescents with type 1 diabetes mellitus in addition to 17 healthy children at the Pediatric Endocrinology Unit, Pediatric Department, Zagazig University Hospitals, from June 2020 to April 2021. All patients were subjected to laboratory investigations including fasting blood glucose levels, HbA1c, kidney function tests, lipid profile, serum albumin, urinary albumin, albumin creatinine ratio, glomerular filtration rate (GFR) and serum preptin.

Results: This study showed that age, weight and BMI were significantly higher among group II with no significant difference between group I and II by LSD. Also, there was no significant difference among groups regarding gender or family history of DM.

Conclusion: Serum preptin is significantly lower in patients with diabetic nephropathy compared with diabetic patients without nephropathy and healthy subjects.
\end{abstract}

Keywords: Preptin, Type-1 Diabetes Mellitus, Diabetic nephropathy.

\section{INTRODUCTION}

Type 1 diabetes mellitus (T1DM) is a chronic autoimmune disease characterized by increased blood glucose levels (hyperglycemia), which are due to insulin deficiency that occurs as the consequence of the loss of the pancreatic islet $\beta$-cells ${ }^{(\mathbf{1}, \mathbf{2})}$. Type 1 diabetes is one of the most prevalent long-term diseases of childhood globally. It has major health consequences for individuals and society. Numerous studies have reported an increasing incidence of Type 1 diabetes in children worldwide, with significant variation in incidence by country ${ }^{(3)}$.

The complications of chronic diabetes are subdivided into microvascular and macrovascular complications. Microvascular complications include nephropathy, neuropathy and retinopathy, which are specific to diabetes. Macrovascular complications manifest predominantly as coronary heart disease, but also cerebrovascular disease and peripheral artery disease; these conditions are not specific to diabetes, but people with T1DM are at risk of developing these conditions ${ }^{(4)}$. Diabetic nephropathy (DN), a chronic and progressive process leading to end-stage renal failure, has a prevalence of approximately $20 \%-40 \%$ in patients with diabetes. Metabolic and hemodynamic alterations caused by hyperglycemia and hypertension are considered to be regulators in the pathogenesis of $\mathrm{DN}^{(5)}$. There are no effective treatments for DN except delaying the progression of $\mathrm{DN}$ or renal replacement therapies. Hence, it is essential to look for new biomarkers for early diagnosis and perform some reasonable treatments for patients at risk ${ }^{(6)}$.
Insulin is one of the most important hormones in regulating body metabolism. Insulin secretion and/ or action are affected by several other peptides ${ }^{(7)}$. Preptin is a peptide related to glucose metabolism, which consists of 34 amino acids. Preptin originates from proinsulin-like growth factor II (pro IGF II). It is secreted from pancreatic $\beta$ cells in response to glucose ${ }^{(8)}$. Preptin levels were reported to be altered in patients with type 2 diabetes with glucose intolerance. Besides, preptin was also reported to mediate insulin secretion physiologically depending on glucose concentrations ${ }^{(\mathbf{9})}$. Pancreatic $\beta$-cell destruction in patients with type I diabetes mellitus avoids the secretion of insulin and preptin, whereby falling their effects on the RUNX2 gene ${ }^{(10)}$.

Wnt signaling pathway plays an important role in podocyte dysfunction of DN ${ }^{(\mathbf{1 1 1})}$. Xiao et al. ${ }^{(\mathbf{1 2})}$ reported that preptin promoted the proliferation and osteogenesis of osteoblast-like cells by activating Wnt/ $\beta$-catenin signaling pathway. Therefore, preptin may promote the podocyte dysfunction, the progression of albuminuria, and at last DN through $\mathrm{Wnt} / \beta$-catenin pathway ${ }^{(6)}$.

This study aimed to evaluate a new biomarker such as preptin in patients with type 1 diabetes and to assess its relation to diabetic nephropathy.

\section{PATIENTS AND METHODS}

A total of 34 children and adolscents selected from the Pediatric Diabetes Clinic were included in the study in addition to 17 healthy children. 
Each of the eligible children was randomly assigned by simple randomization to Group I, Group II or Group III:

Group I: consisted of 17 children recently diagnosed with type 1 diabetes (less than 5 years).

Group II: consisted of 17 children previously diagnosed with type 1 diabetes for more than five years and further stratified to group complicated with diabetic nephropathy and group have no diabetic nephropathy.

Group III (control group): consisted of 17 healthy children without any infection or chronic disease, with no family history and of age- and sex- matched with diabetic children.

\section{Ethical approval:}

The study protocol was revised and approved by the Committee of Research Ethics of Faculty of Medicine, Zagazig University. The work has been carried out in accordance with The Code of Ethics of the World Medical Association (Declaration of Helsinki) for studies involving humans. Prior to participation, all caregivers of eligible children were educated about the study protocol and signed a written informed consent.

\section{Inclusion criteria:}

Patients with type 1 diabetes diagnosed according to the criteria for diagnosis of diabetes. Patients under the age of 18 years. Active diabetic nephropathy diagnosed in the form of microalbuminuria (urinary albumin excretion [UAE] 30-299 mg/g creatinine in two of three samples over a 3- to 6- months period despite angiotensin converting enzyme inhibitors) ${ }^{(13)}$.

\section{Exclusion criteria:}

Patients unable or unwilling to give informed consent. Patients during acute diabetic complications, for example, diabetic ketoacidosis (DKA) or hypoglycemia. Patients with urinary tract infections, urolithiasis, liver cirrhosis, cardiac disease, congestive heart failure, overt proteinuria, or other known major diseases. Patients on long term steroids, and chemotherapy.

All patients were subjected to detailed medical history with special emphasis on demographic data (age, sex, race, ethnicity, gender, income). Complete examination including chest, heart, abdomen and especial emphasis on nephrological examination for evidence of any diabetic complications. Investigations included measurement of fasting blood glucose levels using Cobas Integra 800. HbA1c\% using D-10. Routine kidney function tests including serum creatinine and
BUN using Cobas Integra 800. Measurement of lipid profile and serum albumin using Cobas Integra 800. Urinary albumin excretion (UAE) in an early morning fasting urine sample as albumin-to-creatinine ratio (UACR) by an immuno-turbidimetric method. Albumin creatinine ratio. Glomerular filtration rate (GFR) according to MDRD. Serum preptin was measured by enzyme linked immunosorbent assay (ELISA) kit by Sun Red Biotech Co., Ltd (China).

\section{Measurement of preptin:}

The Human preptin (Enzyme-Linked Immunosorbent Assay) kit is designed for quantitative measurement of preptin level in the sample. Add preptin to monoclonal antibody enzyme well, which is precoated with human preptin monoclonal antibody incubation. Then, add preptin antibodies labeled with biotin, and combined with Streptavidin-HRP to form immune complex. Then, incubation and washing again to remove the uncombined enzyme. Then, add chromogen solution A and B, the color of the liquid changes into the blue, and at the effect of acid, the color finally becomes yellow. The chroma of color and the concentration of the human substance preptin of sample were positively correlated.

\section{Statistical Analysis}

Data collected throughout history, basic clinical examination, laboratory investigations and outcome measures were coded, entered and analyzed using Microsoft Excel software. Data were then imported into statistical package for the social sciences (SPSS version 20.0) software for analysis. According to the type of data, qualitative were presented as number and percentage and quantitative continues group data were presented by mean $\pm \mathrm{SD}$. The following tests were used to test differences for significance: Difference and association of qualitative variable by Chi square test $\left(\mathrm{X}^{2}\right)$. Differences between quantitative independent groups by $\mathrm{t}$ test or Mann Whitney, multiple by ANOVA or Kruskal Wallis and correlation by Pearson's correlation or Spearman's, logistic regression for independent predictors. $\mathrm{P}$ value $\leq 0.05$ for significant results $\&<0.001$ for highly significant result.

\section{RESULTS}

Table (1) showed that age was distributed as 10.23 $\pm 2.63,13.58 \pm 2.34$ and $11.23 \pm 3.01$ and group II was significantly higher regarding age. Also, weight and BMI were significantly higher among group II with no significant difference between group I and II by LSD. Besides, there was no significant difference among groups regarding gender or family history of DM. 
Table (1): Basic demographic and anthropometric measures among studied groups

\begin{tabular}{|c|c|c|c|c|c|c|c|}
\hline & & & $\begin{array}{c}\text { Group I } \\
(\text { no }=17)\end{array}$ & $\begin{array}{l}\text { Group II } \\
(\text { no }=17)\end{array}$ & $\begin{array}{l}\text { Group III } \\
(\text { no }=17)\end{array}$ & $\mathbf{F} / \mathbf{X}^{2}$ & $\mathbf{P}$ \\
\hline \multicolumn{3}{|c|}{$\begin{array}{c}\text { Age (years) } \\
\text { Mean } \pm \text { SD }\end{array}$} & $10.23 \pm 2.63$ & $13.58 \pm 2.34 *$ & $11.23 \pm 3.01$ & 7.024 & $0.002 *$ \\
\hline \multicolumn{3}{|c|}{$\begin{array}{l}\text { Weight (kg) } \\
\text { Mean } \pm \text { SD }\end{array}$} & $31.35 \pm 8.54$ & $41.94 \pm 10.9 *$ & $34.88 \pm 8.9$ & 5.462 & $0.007 *$ \\
\hline \multicolumn{3}{|c|}{$\begin{array}{l}\text { Height }(\mathrm{m}) \\
\text { Mean } \pm \text { SD }\end{array}$} & $1.37 \pm 0.1$ & $1.46 \pm 0.09$ & $1.40 \pm 0.12$ & 3.333 & 0.044 \\
\hline \multicolumn{3}{|c|}{$\begin{array}{c}\text { BMI }\left(\mathrm{kg} / \mathrm{m}^{2}\right) \\
\text { Mean } \pm \text { SD }\end{array}$} & $16.31 \pm 1.94$ & $19.16 \pm 3.05^{*}$ & $17.34 \pm 1.87$ & 6.386 & $0.003 *$ \\
\hline \multirow{4}{*}{ Gender } & \multirow{2}{*}{ Female } & $\mathbf{N}$ & 8 & 8 & 8 & \multirow{4}{*}{0.00} & \multirow{4}{*}{1.00} \\
\hline & & $\%$ & $47.1 \%$ & $47.1 \%$ & $47.1 \%$ & & \\
\hline & \multirow{2}{*}{ Male } & $\mathbf{N}$ & 9 & 9 & 9 & & \\
\hline & & $\%$ & $52.9 \%$ & $52.9 \%$ & $52.9 \%$ & & \\
\hline \multirow{4}{*}{$\begin{array}{c}\text { Family history } \\
\text { of DM }\end{array}$} & \multirow{2}{*}{ No } & $\mathbf{N}$ & 14 & 13 & 17 & \multirow{4}{*}{2.33} & \multirow{4}{*}{0.31} \\
\hline & & $\%$ & $82.4 \%$ & $76.5 \%$ & $100.0 \%$ & & \\
\hline & \multirow{2}{*}{ Yes } & $\mathbf{N}$ & 3 & 4 & 0 & & \\
\hline & & $\%$ & $17.6 \%$ & $23.5 \%$ & $0.0 \%$ & & \\
\hline
\end{tabular}

* Group cause significant

Table (2) showed that Preptin was significantly lower among group II than Group I and significantly higher at group III.

Table (2): Preptin distribution among studied groups

\begin{tabular}{|c|c|c|c|c|c||}
\hline & $\begin{array}{c}\text { Group I } \\
(\mathbf{n}=\mathbf{1 7})\end{array}$ & $\begin{array}{c}\text { Group II } \\
(\mathbf{n}=\mathbf{1 7})\end{array}$ & $\begin{array}{c}\text { Group III } \\
(\mathbf{n = 1 7})\end{array}$ & $\begin{array}{c}\text { F/ Kruskal } \\
\text { Walis }\end{array}$ & P \\
\hline \hline $\begin{array}{c}\text { Preptin } \\
(\mathbf{m g} / \mathbf{m l}) \\
\text { Mean } \mathbf{\text { SD }}\end{array}$ & $103.17 \pm 9.15 \#$ & $75.88 \pm 7.27 \#$ & $147.47 \pm 9.34 \#$ & 296.985 & $0.00^{* *}$ \\
\hline
\end{tabular}

* Group cause significant

Table (3) showed that diabetic nephropathy was significantly associated with group II with $15 / 17$ cases (88.2\%) then with group I [4/17 cases (23.5\%)] while no cases found in control.

Table (3): Nephropathy distribution among studied Groups

\begin{tabular}{|c|c|c|c|c|c|c|c|}
\hline & & & & Group & & & \\
\hline & & & $\begin{array}{l}\text { Group I } \\
(\text { no }=17)\end{array}$ & $\begin{array}{l}\text { Group II } \\
(\text { no= 17) }\end{array}$ & $\begin{array}{c}\text { Group III } \\
(\text { no= }=17)\end{array}$ & $\mathbf{X}^{2}$ & $\mathbf{P}$ \\
\hline \multirow{4}{*}{$\begin{array}{c}\text { Diabetic } \\
\text { Nephropathy }\end{array}$} & \multirow{2}{*}{ No } & $\mathbf{N}$ & 13 & 2 & 17 & \multirow{4}{*}{30.3} & \multirow{4}{*}{$0.00 * *$} \\
\hline & & $\%$ & $76.5 \%$ & $11.8 \%$ & $100.0 \%$ & & \\
\hline & \multirow{2}{*}{ Yes } & $\mathbf{N}$ & 4 & 15 & 0 & & \\
\hline & & $\%$ & $23.5 \%$ & $88.2 \%$ & $0.0 \%$ & & \\
\hline
\end{tabular}

Figure (1) showed significant AUC with cutoff $<122$ with sensitivity $100 \%$ and specificity $100 \%$. 


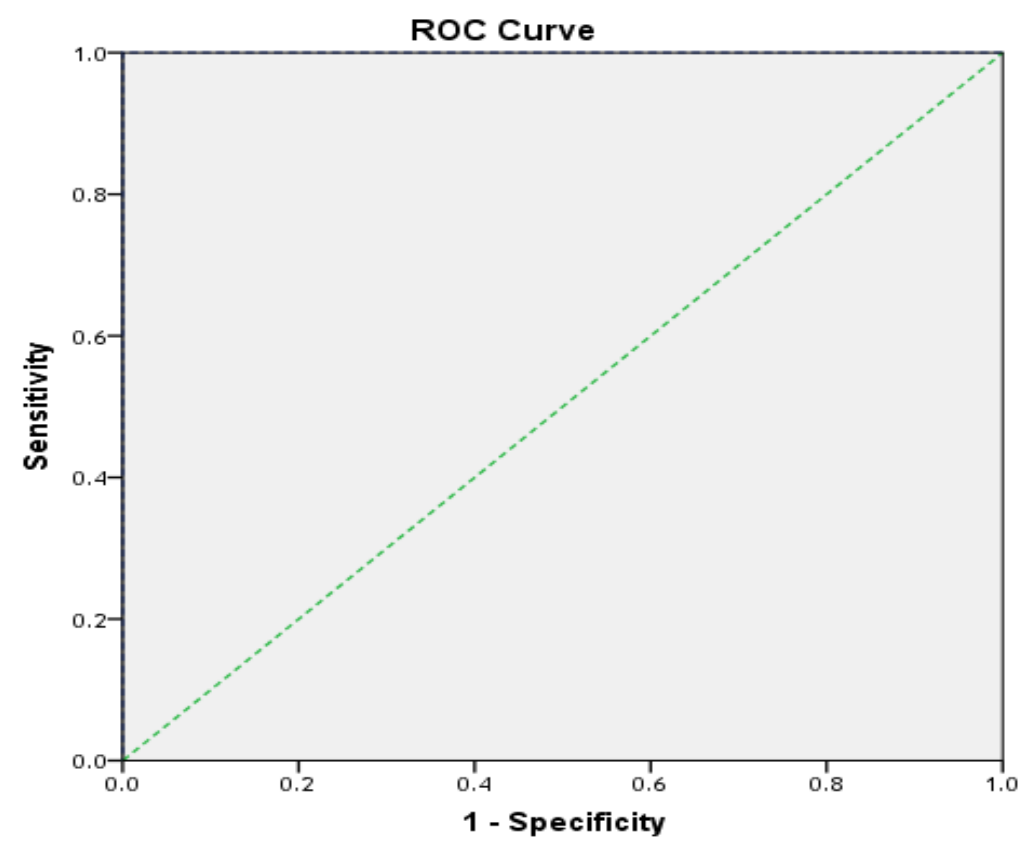

Figure (1): ROC Curve for detection of diabetic cases cutoff regard Preptin

Table (4) showed that nephropathy cases were significantly higher regarding age, BMI, duration of diabetes, FBS, cholesterol, triglycerides, creatinine, BUN, urinary albumin and ACR and significantly lower regarding HDL, GFR and preptin.

Table (4): Comparison between nephropathy cases and non-nephropathy cases to detect predictors of nephropathy

\begin{tabular}{|c|c|c|c|c|c|c|}
\hline & & & $\begin{array}{c}\text { No diabetic } \\
\text { nephropathy } \\
(\text { no }=32)\end{array}$ & $\begin{array}{c}\text { Diabetic } \\
\text { nephropathy } \\
(\text { no }=19) \\
\end{array}$ & $\begin{array}{l}\text { t/ Mann } \\
\text { Whitney }\end{array}$ & $\mathbf{P}$ \\
\hline \multicolumn{3}{|c|}{ Age (years) } & $10.33 \pm 3.17$ & $13.15 \pm 2.19$ & 3.065 & $0.004 *$ \\
\hline \multicolumn{3}{|c|}{ Weight (kg) } & $32.60 \pm 11.95$ & $39.84 \pm 9.37$ & 1.981 & 0.056 \\
\hline \multicolumn{3}{|c|}{ Height (M) } & $1.38 \pm 0.13$ & $1.45 \pm 0.08$ & 1.902 & 0.066 \\
\hline \multicolumn{3}{|c|}{ BMI $\left(\mathrm{kg} / \mathrm{m}^{2}\right)$} & $16.52 \pm 2.54$ & $18.69 \pm 2.87$ & 2.287 & $0.029 *$ \\
\hline \multicolumn{3}{|c|}{ Age of Onset of DM (years) } & $7.73 \pm 2.68$ & $6.47 \pm 2.34$ & 1.460 & 0.154 \\
\hline \multicolumn{3}{|c|}{ Duration of Diabetes } & $2.8 \pm 0.94$ & $6.31 \pm 2.3$ & 4.756 & $0.00 * *$ \\
\hline \multicolumn{3}{|c|}{ SBP ( mmhg) } & $118.26 \pm 6.39$ & $120.63 \pm 8.78$ & 0.874 & 0.389 \\
\hline \multicolumn{3}{|c|}{ DBP ( mmhg) } & $73.80 \pm 4.22$ & $73.80 \pm 7.67$ & 0.052 & 0.959 \\
\hline \multicolumn{3}{|c|}{ FBS $(\mathrm{mmol} / \mathrm{L})$} & $175.0 \pm 4.28$ & $209.57 \pm 21.69$ & 3.094 & $0.004 *$ \\
\hline \multicolumn{3}{|c|}{ HBA1C (gm\%) } & $8.60 \pm 1.53$ & $9.49 \pm 1.69$ & 1.594 & 0.121 \\
\hline \multicolumn{3}{|c|}{ Cholesterol (mg/dl) } & $175.46 \pm 7.19$ & $182.63 \pm 8.18$ & 2.670 & $0.012 *$ \\
\hline \multicolumn{3}{|c|}{ Triglycerides } & $122.06 \pm 7.67$ & $131.15 \pm 9.03$ & 3.108 & $0.004 *$ \\
\hline \multicolumn{3}{|c|}{ HDL (mg/dl) } & $37.60 \pm 4.73$ & $33.26 \pm 5.66$ & 2.379 & $0.023 *$ \\
\hline \multicolumn{3}{|c|}{ LDL (mg/dl) } & $108.40 \pm 5.70$ & $111.89 \pm 7.12$ & 1.547 & 0.132 \\
\hline \multicolumn{3}{|c|}{ Creat (mg/dl) } & $0.95 \pm 0.14$ & $1.08 \pm 0.13$ & 2.607 & $0.014^{*}$ \\
\hline \multicolumn{3}{|c|}{ BUN (mg/dl) } & $18.93 \pm 3.04$ & $23.31 \pm 6.29$ & 2.193 & $0.036^{*}$ \\
\hline \multicolumn{3}{|c|}{ Serum albumin (g/dl) } & $4.34 \pm 0.48$ & $4.26 \pm 0.52$ & 0.441 & 0.662 \\
\hline \multicolumn{3}{|c|}{ Urinary albumin $(\mathrm{mcg} / \mathrm{ml})$} & $29.31 \pm 3.85$ & $113.43 \pm 3.2$ & 4.730 & $0.00^{* * *}$ \\
\hline \multicolumn{3}{|c|}{$\mathrm{ACR}(\mathrm{mg} / \mathrm{dl})$} & $21.65 \pm 2.96$ & $104.65 \pm 7.3$ & 15.102 & $0.00 * *$ \\
\hline \multicolumn{3}{|c|}{ GFR (mg/dl) } & $115.38 \pm 6.50$ & $52.46 \pm 1.55$ & 14.365 & $0.00 * *$ \\
\hline \multicolumn{3}{|c|}{ Preptin (mg/dl) } & $101.46 \pm 12.73$ & $80.10 \pm 11.60$ & 5.106 & $0.00 * *$ \\
\hline \multirow{4}{*}{$\begin{array}{c}\text { Family } \\
\text { history of } \\
\text { DM }\end{array}$} & & $\mathbf{N}$ & 11 & 16 & \multirow{4}{*}{0.607} & \multirow{4}{*}{0.43} \\
\hline & No & $\%$ & $73.3 \%$ & $84.2 \%$ & & \\
\hline & & $\mathbf{N}$ & 4 & 3 & & \\
\hline & Yes & $\%$ & $26.7 \%$ & $15.8 \%$ & & \\
\hline \multirow{2}{*}{\multicolumn{2}{|c|}{ Total }} & $\mathbf{N}$ & 15 & 19 & & \\
\hline & & $\%$ & $100.0 \%$ & $100.0 \%$ & & \\
\hline
\end{tabular}


Table (5) showed that ACR, GFR and preptin were the only independent predictors for nephropathy.

Table (5): Multivariate logistic regression for independent predictors of nephropathy in patients with diabetes mellitus

\begin{tabular}{|c|c|c|c|c|c|}
\hline & \multirow{2}{*}{ Wald } & \multirow{2}{*}{ Sig. } & \multirow{2}{*}{ OR } & \multicolumn{2}{|c|}{ 95\% C.I } \\
\cline { 5 - 6 } & & & & Lower & Upper \\
\hline \hline Age (years) & 1.854 & 0.125 & 1.236 & 0.951 & 10.254 \\
\hline BMI (kg/m $\mathbf{2})$ & 1.466 & 0.225 & 2.581 & 0.852 & 11.258 \\
\hline Duration of Diabetes & 2.085 & 0.098 & 1.859 & 0.965 & 6.669 \\
\hline FBS (mmol/l) & 2.123 & 0.087 & 3.074 & 0.741 & 14.854 \\
\hline Cholesterol (mg/dl) & 1.062 & 0.485 & 1.982 & 0.884 & 6.9654 \\
\hline Triglycerides & 1.745 & 0.144 & 9.819 & 0.963 & 19.639 \\
\hline HDL (mg/dl) & 1.368 & 0.305 & 2.145 & 0.744 & 17.6321 \\
\hline Creatnine (mg/dl) & 2.255 & 0.081 & 5.236 & 0.855 & 9.6959 \\
\hline BUN (mg/dl) & 2.145 & 0.091 & 3.102 & 0.988 & 8.6987 \\
\hline Urinary albumin (mcg/ml) & 2.321 & 0.073 & 8.464 & 0.815 & 11.856 \\
\hline ACR (mg/dl) & 4.854 & $0.00^{* *}$ & 12.042 & 2.523 & 16.398 \\
\hline GFR (mg/dl) & 3.874 & $0.0002^{* *}$ & 16.097 & 3.258 & 20.365 \\
\hline Preptin (mg/dl) & 3.774 & $0.0005 * *$ & 11.028 & 1.476 & 15.245 \\
\hline
\end{tabular}

Table (6) showed significant AUC with cutoff < 93.5 with sensitivity $80.0 \%$ and specificity $83.3 \%$.

Table (6): Area under curve, cutoff and validity for suggested cutoff regard preptin for detection of nephropathy

\begin{tabular}{|c|c|c|c|c|c|c|}
\hline \multirow{2}{*}{ Area } & \multirow{2}{*}{ Cutoff } & \multirow{2}{*}{$\mathbf{P}$} & \multicolumn{2}{|c|}{$\mathbf{9 5 \% \text { Confidence Interval }}$} & \multirow{2}{*}{ Sensitivity } & \multirow{2}{*}{ Specificity } \\
\cline { 4 - 5 } & & $\begin{array}{c}\text { Lower } \\
\text { Bound }\end{array}$ & Upper Bound & & \\
\hline \hline 0.881 & $<93.5$ & $0.00^{* *}$ & 0.761 & 1.000 & $80.0 \%$ & $83.3 \%$ \\
\hline
\end{tabular}

\section{DISCUSSION}

This study regarding gender showed that the male to female ratio was $1: 1$, this is because type 1 diabetes mellitus is a genetic disease equally affect male and female. This finding is in agreement with studies done by Kalyva et al. ${ }^{(14)}$ and Emmanouilidou et al. ${ }^{(15)}$ in Greece. Also, Abd El Dayem et al. ${ }^{(9)}$ found that there was no significant difference between the different groups regarding gender with patients with type 1 diabetes (50\% males and 50\% females) and healthy volunteers (50\% males and 50\% females).

In the present study, weight and BMI were significantly higher in DN group than control group and T1DM without DN. This is in agreement with study of Dawood et al. ${ }^{(16)}$ who found that BMI was significantly higher in DN groups compared with the control group. In disagreement with our study, Jabar ${ }^{(17)}$ found that there was no significant difference concerning the body mass index between the patients $\left(24.31 \pm 0.54 \mathrm{~kg} / \mathrm{cm}^{2}\right)$ and the control $\left(26.18 \pm 0.57 \mathrm{~kg} / \mathrm{cm}^{2}\right)$. This difference may be because of the species differences and differences in study design.

In the current study, family history of DM was positive in $17.6 \%$ and $23.5 \%$ in group I and II. This comes in agreement with Hjort $\boldsymbol{e t}$ al. ${ }^{\mathbf{( 1 8 )}}$ who found that family history of diabetes is a strong predictor of diabetes risk and that previous studies have indicated that diabetes in first-degree relatives confers a nine-fold greater risk of type 1 diabetes.
In the current study, T1DM and DN groups showed significantly decreased serum preptin concentrations compared to the control group. Moreover, serum preptin concentrations were significantly decreased in DN group compared to those with T1DM group. Low preptin level in TIDM patient might be resulting from decreased preptin secretion associated with decreased insulin secretion in T1DM patient.

In present study, diabetic nephropathy patient were significantly higher in group II with $15 / 17$ cases $(88.2 \%)$ then in group I with $4 / 17(23.5 \%)$, while there were no cases found in control group. This indicate that the longer duration of T1DM the higher incidence of DN to occur.

The validity for suggested cutoff regarding preptin for detection of diabetes showed significant AUC with cutoff < 122 with sensitivity $100 \%$ and specificity $100 \%$ (figure 1). This comes in agreement with Jabar ${ }^{(17)}$ who found that the children affected with type- 1 diabetes mellitus had a significantly lower serum level of preptin hormone $(82.79 \pm 4.3 \mathrm{ng} / \mathrm{ml})$ compared to the control group $(138.62 \pm 6.1 \mathrm{ng} / \mathrm{ml})$ at a $\mathrm{p}$ value fewer than 0.05 . Also, Wang et al. ${ }^{(19)}$ found that the preptin was significantly lower in the diabetics than controls.

Pancreatic islet beta-cells secrete the preptin along with insulin and amylin. Preptin may enhance the insulin-like growth factor receptor linked to the protein kinase to enhance the calcium-dependent insulin secretion under great glucose levels. This result may 
provide a new insight about the autocrine action of preptin ${ }^{(20)}$. It had been suggested that unstable blood glucose concentrations similar to those seen during the postprandial hyperglycemic condition in people with type I-DM may contribute to a significant oxidative stress - even more than that seen in chronically elevated blood glucose (21). Dysfunction of the mitochondria could be one of many chief underlying imperfections that linking obesity to type I diabetes, by decreasing insulin sensitivity and by compromising $\beta$-cell function of pancreas. The results clears that there is a negative correlation between preptin hormone level and glucose level in patients group. Preptin level may be decreased with insulin level decreasing therefore it decreases with diabetic patients and increases with control group with increasing insulin concentration ${ }^{(22)}$.

In disagreement with our study, an investigation performed in patients with type 1 diabetes showed that patients with type 1 diabetes had significantly increased preptin concentrations than in the controls ${ }^{(9)}$. In addition, gestational diabetes mellitus (GDM) patients had elevated plasma, cord blood, and colostrum preptin than the control women ${ }^{(23)}$. However, another study demonstrated that there was no statistical difference in preptin between GDM patients and controls ${ }^{(\mathbf{8})}$. As for the status of prediabetes, impaired glucose tolerance (IGT) subjects had elevated serum preptin than subjects with normal glucose tolerance ${ }^{(24)}$. However, Yang et al. (7) reported that serum preptin concentrations showed no significant differences between IGT and healthy controls. These conflicting results may be explained by different enrolled populations and different ELISA kits.

In our study nephropathy cases were significantly higher regarding age, BMI, duration of diabetes, FBS, cholesterol, triglycerides, creatinine, BUN, urinary albumin and ACR and significantly lower regarding HDL and preptin. However, ACR, GFR and preptin were the only independent predictors for nephropathy in this study.

In the present study, preptin is significantly positively correlated with GFR, onset of diabetes, HDL and serum albumin but significantly negatively correlated with ACR, urinary albumin, creatinine, BUN, duration of diabetes, SBP, FBS, cholesterol, triglycerides, LDL, BMI and age. This result demonstrated that decreased serum preptin was correlated with DN and with renal function declines.

The validity for suggested cutoff regarding preptin for detection of nephropathy showed significant AUC with cutoff $<93.5$ with sensitivity $80.0 \%$ and specificity $83.3 \%$. Our result comes in agreement with other studies which found that serum preptin was correlated with metabolic syndrome characteristics including obesity ${ }^{(25,20)}$, hypertension ${ }^{(24,26)}$ and hyperlipidemia ${ }^{(7}$, 24). Also, El-Eshmawy and Abdel Aal. ${ }^{(20)}$ found that preptin was correlated with BMI. This confirms the important role of preptin in body metabolic mechanism.

We found association between preptin and systolic blood pressure which may suggest early renal injury and
DN progression. Furthermore the association between preptin and systolic blood pressure may suggest indirect prediction role of preptin as a cardiovascular morbidity marker in diabetic patient ${ }^{\left({ }^{(6)}\right.}$. However, Yang et al. ${ }^{(7)}$ observed no correlations between serum preptin and diastolic blood pressure, serum triglycerides, and serum HDL-C values. Also, Ahmad et al. ${ }^{(27)}$ found that there was no association noticed between serum preptin levels and indices of lipid profile. Our results disagree with Wang $\boldsymbol{e t}$ al. ${ }^{(\boldsymbol{6})}$ who found that increased serum preptin concentrations were correlated with DN and renal functional parameters this difference may be due to that their study was on T2DM in adults.

\section{CONCLUSION}

Serum preptin is significantly lower in patients with diabetic nephropathy compared to diabetic patients without nephropathy and healthy subjects.

We recommend further studies on large sample size to understand the complicated signaling pathways as well as physiological significance of preptin.

\section{REFERENCES}

1. Atkinson M, Eisenbarth G, Michels A (2014): Type 1 diabetes. Lancet, 383: 69-82.

2. Katsarou A, Gudbjörnsdottir S, Rawshani A et al. (2017): Type 1 diabetes mellitus. Nature Reviews Disease Primers, 3 (1): 1-17.

3. Yeşilkaya E, Cinaz P, Andıran N et al. (2017): First report on the nationwide incidence and prevalence of Type 1 diabetes among children in Turkey. Diabetic Medicine, 34 (3): 405- 410.

4. Zimmerman R (2016): Diabetes mellitus: management of microvascular and macrovascular complications. Cleveland Clinic: Centers for Continuing Education. http://www.clevelandclinicmeded.com/medicalpubs/dis easemanagement/endocrinology/diabetes-mellitus/

5. Satirapoj B (2012): Nephropathy in diabetes. Adv Exp Med Biol., 771: 107-22.

6. Wang R, Xue A, Zheng W et al. (2019): Elevated serum preptin concentrations in patients with diabetic nephropathy. Journal of Investigative Medicine, 67 (7): 1048-1052.

7. Yang G, Li L, Chen W et al. (2009): Circulating preptin levels in normal, impaired glucose tolerance, and type 2 diabetic subjects. Ann Med., 41: 52-6.

8. Baykus Y, Gurates B, Aydin S et al. (2012): Changes in serum obestatin, preptin and ghrelins in patients with Gestational Diabetes Mellitus. Clin Biochem., 45: 198202.

9. Abd El Dayem S, Battah A, El Shehaby A et al. (2015): Assessment of human cartilage glycoprotein 39 (YKL-40), preptin, and nitric oxide in adolescent patients with type 1 diabetes and its relation to cardiorenal affection. J Pediatr Endocrinol Metab., 28: 309-14.

10. Christine H, Stephan K, Günther $K$ et al. (2012): Bone, sweet bone-osteoporotic fractures in diabetes mellitus. Nature Reviews Endocrinology, 8: 297-305.

11. Dai H, Liu Q, Liu B (2017): Research progress on mechanism of podocyte depletion in diabetic nephropathy. J Diabetes Res., 17: 1-10. 
12. Xiao C, Li W, Lu T et al. (2019): Preptin Promotes Proliferation and Osteogenesis of MC3T3-E1 Cells by Upregulating $\beta$-Catenin Expression. IUBMB Life, 71: 854-862.

13. Molitch M, DeFronzo R, Franz M et al. (2004): Nephropathy in diabetes. Diabetes Care, 27: 79-83.

14. Kalyva E, Malakonaki E, Rinard S (2011): Health related quality of life in children with type 1 diabetes mellitus: self and parenteral perceptions. Pediatric Diabetes, 12: 34-40

15. Emmanouilidou E, Karavatos A, Crist D et al. (2008): Quality of life in children and adolescents with diabetes of Northern Greek origin. Hippokratia, 3: 168175.

16. Dawood A, Abdelraof M, EI Ghobashy Y (2017): The relationship between serum apelin level and different grades of diabetic nephropathy in type 2 diabetic patients. Egyptian Journal of Obesity, Diabetes and Endocrinology, 3 (1): 32-36.

17. Jabar H (2019): Serum Level of Preptin Hormone in Children with Type-1 Diabetes Mellitus. Medical Journal of Tikrit University, 25 (1): 115-122.

18. Hjort $R$, Alfredsson $L$, Andersson $T$ et al. (2017): Family history of type 1 and type 2 diabetes and risk of latent autoimmune diabetes in adults (LADA). Diabetes \& Metabolism, 43 (6): 536-542.

19. Wang H, Li Y, Gao X et al. (2012): The Association between Chronic Complications of Type 2 Diabetic and Anti-Oxldl and Preptin [J]. Progress in Modern Biomedicine, 12: 25-32.
20. El-Eshmawy M, Abdel Aal I (2015): Relationships between preptin and osteocalcin in obese, overweight, and normal weight adults. Appl Physiol Nutr Metab., 40: 218-22.

21. Hirsh I, Brownlee M (2005): Should minimal blood glucose variability become the gold standard of glycemic control? J Diabetes Complications, 19: 17881 .

22. Robert H, Eckel M, Steven E et al. (2011): Obesity and Type 2 Diabetes: What Can Be Unified and What Needs to Be Individualized? Diabetes Care, 34: 14241425.

23. Aslan M, Celik O, Karsavuran N et al. (2011): Maternal serum and cord blood preptin levels in gestational diabetes mellitus. J Perinatol., 31: 350-5.

24. Bu Z, Kuok K, Meng J et al. (2012): The relationship between polycystic ovary syndrome, glucose tolerance status and serum preptin level. Reprod Biol Endocrinol., 10: $10-14$.

25. Ozkan Y, Timurkan E, Aydin S et al. (2013): Acylated and desacylated ghrelin, preptin, leptin, and nesfatin-1 Peptide changes related to the body mass index. Int J Endocrinol., 13: 1-7.

26. Cai H, Liu Q, Dong X et al. (2018): Plasma preptin levels are decreased in patients with essential hypertension. Pharmazie, 73: 274-8.

27. Ahmad S, Nourollahi S, Nakhjavani M et al. (2019). Preptin and Myostatin Independently Increase in PreDiabetics and Patients of Type 2 Diabetes Mellitus. Acta Medica Iranica, 31: 160-166. 